union; very oblique fractures with tendency to displacement, permanent interposition between fragments of soft parts, insufficient fixation of fragments, etc. The cases which were to be ununited fractures always showed no tendency to union, never merely a yielding of fragment. The results showed great impairment of function. Callus formation was entirely absent in nine; in the other five a mass of connective tissue united the fragments. The duration of these cases from time of injury to treatment was as follows: six within six months; seven, from seven to eleven and one-quarter months; one, two and one-half years. At the operation, isolated cicatrized ends of fragments were found in eight cases; in three others, a fibrous union of fragments. No case of neïrthrosis was reported. Mechanical treatment was unsuccessful except in one case, and here the substance between the fragments was subcutaneously ruptured.

The operative treatment: In two cases, nails in the connective tissue band of union (Malgaigne); once, no reaction; once, slight fever, marked local pain, subsequent consolidation; in four cases, the Diefferbach method, with the modification of cutting off the ivory pegs at the surface of the bone and leaving them permanently in situ (Bidder); resection of ends of fragments in eight cases; six times with silver-wire suture. Seven successful results. One case remained unhealed and refused a second operation. In one case, a femur, the upper fragment was cut wedge-shaped, and inserted in the central cavity of the lower.

In this connection Munk's ${ }^{89}$ brochure is interesting, in which he reports the successful treatment of five cases (one a fresh compound fracture, four cases of pseudo-arthrosis), by ivory plugs placed in the medullary canals, - one humerus, one femur and three tibiæ. 'Three were discharged with firm union; two with union, but still under observation at time of publication. He attributes the result to the fixation of the fragments by the plug, and to the production of osteoplastic inflammation by its presence. The method seems to him to be especially valuable where there is a separation of fragment ends by loss from comminution or other causes, the plug then forming a firm bridge and being the nucleus for callus formation. This fixation is valuable in fresh oblique fractures of the leg, Munk exposes the ends of the fragments, and then fits the ivory plug into the canal, which is previously bored out, if necessary, to admit it. The technique by which the idory plug is prepared or sterilized is not stated, but the aseptic healing of the wound is considered of vital importance. 'The plug is not absorbed, but remains indefinitely.

(To be continued.)

THe Journal of the American Medical Association (of April 11th) prints a long letter from a correspondent, suggesting certain improvements in that periodical, and giving his reasons in full. He says, in the first place, that "It should be printed on plain and not on glazed paper, which from its reflection, dazzles the eye, irritates the optic nerves, muscles and lobes, brain and mind, and sympathetically other parts of the system, thus impairing the sight, caysing eye-strain with spasm and twitching of muscles, ocular and cerebral hyperæmia, dizziness, headache, neuralgia, melaucholy, petulance, irascibility, and other disorders of the physical, mental and moral nature, more or less serious."

a Boltrag. z. Olin. Chir., 1890, vi, 3, p. 679

\section{Ulinical \&Department.}

\author{
INJURY TO THE EAR FROM A PIECE OF \\ WOOD. ${ }^{1}$ \\ BY FREDERICK L. JACK, M.D.
}

The following brief clinical history of a case of injury to the ear is presented to the Society, chiefly because the case is a very unusual one.

A. D., five years of age, was brought to the Massachusetts Charitable Eye and Ear Infirmary, October 1, 1890, during the service of Dr. Green, to obtain relief from intense pain in the right ear. According to his mother's statement, the cause of the trouble at that time dated back three months. The patient was seen running away from his playmates with a paper wind mill on a stick protruding from the mouth. He stumbled, fell and was carried across the street to his home, bleeding from the mouth, nose and right ear. In the evening the ear became painful. On the following night the pain was intense and extended over the right side of the head. On the next morning a discharge from the ear was noticed.

Since then the pain and otorrhoea had continued. Five weeks after the accident pus began to flow from a spontaneous opening over the tip of the mastoid process. A few days later facial paralysis was noticed. I saw the patient on October 1st, when the condition was as follows: The supra- and retro-auricular regions decidedly swollen. Complete facial paralysis. External auditory meatus filled with granulations. Otorrhœa profuse, also free discharge of pus from the opening over the mastoid. Hearing greatly impaired. 'Iunning-fork placed on vertex heard loudest in the right ear. Examination of the throat negative. Pain for the last ten nights had been very severe, and he was considerably emaciated. Under ether, I enlarged the post-aural opening, and with the probe was unable to detect dead bone at the depth of nearly one inch and a half along the posterior wall of the external canal. The granulations in the external meatus wero removed by means of the suare and forceps. The bleeding rendered a view of the drum membrane impossible, but with the probe what was supposed to be a sequestrum was detected at the bottom of the canal. Efforts to extract it with forceps failed, but it wus easily brought away by means of a hook. The object proved to be a piece of wood, in appearance uearly like one-half of a common brimstone match, which was forced in some way through the membrane of Shrapnel at the time of the fall on the sidewalk three months before.

The patient remained under observation for three weeks, during which time there was no pain, and the ear healed rapidly. On October 20th there was no discharge from the ear, or from the opening over the mastoid. Ou inspection, the external canal was perfectly free from granulations, and the membrane in a cicatricial condition. Hearing for watch twenty inches. Dr. Walton kindly saw the case, and reported "pretty nearly complete facial paralysis with degeneration reaction."

A Tennessev doctor is credited with delivering two children with a pair of shoemuker's pincers, using the curved handles as obstetric forceps.

Read before the Boston Socioty for Medical Observation, Fobruary 2, 1891 . 